
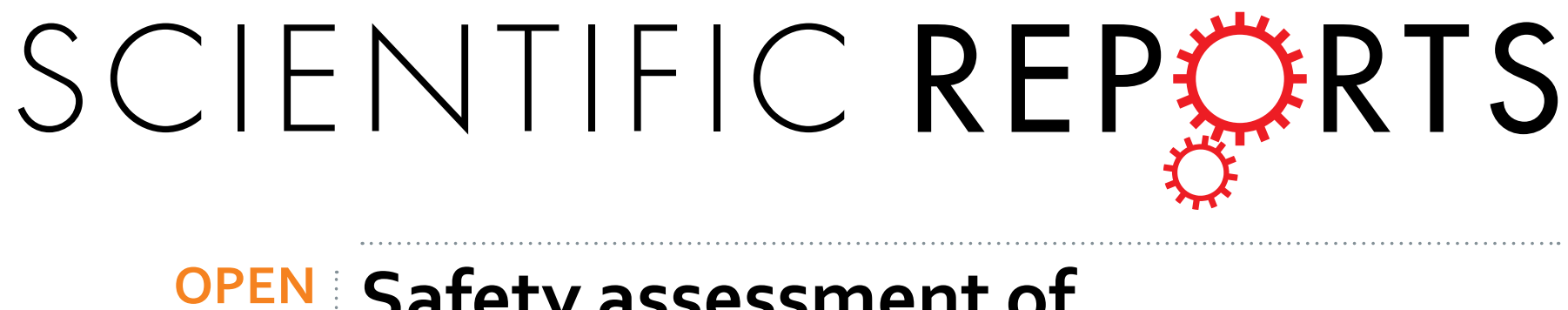

\title{
Safety assessment of
}

Staphylococcus phages of the family Myoviridae based on

Received: 28 June 2016

Accepted: 19 December 2016

Published: 24 January 2017

\section{complete genome sequences}

Zelin Cui ${ }^{1}$, Xiaokui Guo ${ }^{2}$, Ke Dong ${ }^{2}$, Yan Zhang ${ }^{2}$, Qingtian Li ${ }^{3}$, Yongzhang Zhu ${ }^{2}$, Lingbing Zeng ${ }^{2}$, Rong Tang ${ }^{1} \& \mathrm{Li} \mathrm{Li}^{1}$

Staphylococcus phages of the Myoviridae family have a wide host range and potential applications in phage therapy. In this report, safety assessments of these phages were conducted based on their complete genome sequences. The complete genomes of Staphylococcus phages of the Myoviridae family were analyzed, and the Open Reading Frame (ORFs) were compared with a pool of virulence and antibiotic resistance genes using the BLAST algorithm. In addition, the lifestyle of the phages (virulent or temperate) was also confirmed using PHACTS. The results showed that all phages were lytic and did not contain resistance or virulence genes based on bioinformatic analyses, excluding the possibility that they could be vectors for the dissemination of these undesirable genes. These findings suggest that the phages are safe at the genome level. The SceD-like transglycosylase, which is a biomarker for vancomycin-intermediate strains, was widely distributed in the phage genomes. Approximately $70 \%$ of the ORFs encoded in the phage genomes have unknown functions; therefore, their roles in the antibiotic resistance and virulence of Staphylococcus aureus are still unknown and require consideration before use in phage therapy.

Due to the appearance and dissemination of antibiotic-resistant bacteria, concern is increasing that certain bacteria will develop resistance to all known antibiotics, and we will enter a post-antibiotic era. Recently, phage therapy has regained interest among scientists, and some phages have been used in clinical trials with no harmful effects on the trial participants ${ }^{1-3}$.

While phages can be vectors for horizontal gene transfer, several studies have shown that they can also be sources for the transmission of virulence and antibiotic resistance genes among bacteria ${ }^{4}$, thereby accelerating the evolution of virulence and/or antibiotic resistance in bacteria. Phages containing such undesirable genes could be harmful to human health and must be excluded from phage therapy. One study confirmed that Stx production was phage-regulated in $\operatorname{stx}(2 \mathrm{~g})$-positive strains (Shiga toxin-producing Escherichia coli (STEC)) ${ }^{5}$. Report also showed that bacteriophages from poultry, cattle and pigs containing antibiotic resistance genes ${ }^{6}$. These studies suggest that bacteriophages could be environmental vectors for the horizontal transfer of virulence and antibiotic resistance genes.

Staphylococcus phages of the Myoviridae family have been isolated worldwide. Published data show that they have a wide host range and can potentially be used for phage therapy. To date, the complete genome sequences of dozens of Staphylococcus phages of the Myoviridae family have become available, including phage K, G1, Twort, A5W, Sb-1, ISP, SA5, GH15, JD007, SA11, vB_SauM_Remus, vB_SauM_Romulus, S25-3, S25-4, phiIPLA-RODI, phiIPLA-C1C, phiSA012, Team1, P108, MCE-2014, 812, SA1, Staphy1N, MSA6, 676Z, P4W, and Fi200 ${ }^{7-15}$. These phages can potentially be used for the treatment of infectious diseases caused by Staphylococcus aureus in animal models. Phage S25-3 has been shown to prolong life in the silkworm larval infection model and other animal infection models ${ }^{16}$. The Staphylococcus phages Romulus and Remus have infected approximately $70 \%$ of the

${ }^{1}$ Department of Laboratory Medicine, Shanghai General Hospital, Shanghai Jiao Tong University School of Medicine, Shanghai, 200080, China. 'Department of Immunology and Microbiology, School of Medicine, Shanghai Jiao Tong University, Shanghai, 200025, China. ${ }^{3}$ Department of Laboratory Medicine, Ruijin Hospital, Shanghai Jiao Tong University School of Medicine, Shanghai, 200025, China. Correspondence and requests for materials should be addressed to Z.C. (email: czl@sjtu.edu.cn) or L.L. (email: annylish@126.com) 
tested $S$. aureus strains and display promising lytic activity against these isolates ${ }^{15}$. The Staphylococcus phage ISP was shown to be effective against $86 \%$ of tested isolates, including relevant methicillin-resistant S. aureus (MRSA) strains ${ }^{7}$. These studies demonstrate the potential use of phages for the prevention of infectious diseases caused by $S$. aureus. In the present report, a safety assessment based on complete genome sequences of Staphylococcus phages of the Myoviridae family was conducted to evaluate their suitability for clinical use.

\section{Methods}

Complete phage genomes. Staphylococcus phages of the Myoviridae family, including the previously reported phage JD007, had complete genome sequence data. The complete genome sequences were collected from the NCBI database (http://www.ncbi.nlm.nih.gov/genome/viruses/). All Staphylococcus phages belonged to the Myoviridae family and were verified by detailed information provided by the NCBI phylogeny system and their morphology. The published papers related to these phages were also screened. The Staphylococcus phages were confirmed to belong to the Myoviridae family by morphological characterization of the contractile sheath between the head and tail.

General genomic features of the phages. The complete genome sequences of Staphylococcus phages of the Myoviridae family were downloaded in FASTA format from NCBI along with their annotation information. The genomes of the phages were re-annotated using RAST, and each ORF and protein domain search was performed using the InterProScan program and $\mathrm{CDD}^{17,18}$. The phylogeny tree was constructed using MEGA5 with the complete genome sequences based on the neighbour-joining method ${ }^{19}$. The visual alignments of the complete genomes were constructed using the Mauve2.3.1 program $^{20}$.

Lifestyles of the phages. The lifestyles of phages (virulent or temperate) were predicted using the PHACTS program (http://www.phantome.org/PHACTS/index.htm) ${ }^{21}$. The genome annotation results of each phage were analyzed using PHACTS, and their lifestyle was confirmed. PHACTS utilizes a novel algorithm and a supervised Random Forest classifier to predict whether the lifestyle of a phage is virulent or temperate. The algorithm creates a training set from phages with known lifestyles. PHACTS predictions have been shown to have a $99 \%$ precision rate, and PHACTS can also determine the lifestyle of a phage using only genomic data. A current limitation of PHACTS is that a confident lifestyle prediction cannot be made for a small number of phages. This is primarily due to the variability that arises from the random sampling during classifications. If an unknown phage does not have any similarity to phages with known lifestyles in the database, the predictions will be less certain. It is expected that as more phages with known lifestyles are added to the database, the precision rate and sensitivity of the predictions will increase ${ }^{21}$. Additionally, the experimental results of the bacteriophage one step growth curve reported in the corresponding papers were also analyzed to confirm the phage lifestyles.

Analysis of virulence and antibiotic resistance genes. All annotated phage ORFs were used to search an antibiotic resistance gene database (ARDB, http://ardb.cbcb.umd.edu/) ${ }^{22}$ and a virulence factor database (VFDB, http://www.mgc.ac.cn/VFs/main.htm) ${ }^{23}$. Hits with more than $70 \%$ coverage and $30 \%$ identity were considered as positive results. Lihong et al. improved the infrastructural dataset of VFDB: (i) removed the redundancy introduced by previous releases and generated two hierarchical datasets-one core dataset of experimentally verified virulence factors (VFs) only and another full dataset including all known and predicted VFs. Their efforts enhanced the data quality of the VFDB and promoted the usability of the database in the big data era for the bioinformatic mining of the explosively growing data regarding bacterial VFs ${ }^{23}$. Antibiotic Resistance Genes Database (ARDB) - unifying most of the publicly available information on antibiotic resistance. Each gene and resistance type is annotated with rich information, including resistance profile, mechanism of action, ontology, COG and CDD annotations, as well as external links to sequence and protein databases. ARDB database also supports sequence similarity searches and implements an initial version of a tool for characterizing common mutations that confer antibiotic resistance. The information they provided can be used as compendium of antibiotic resistance factors as well as to identify the resistance genes of newly sequenced genes, genomes, or metagenomes ${ }^{22}$.

Phylogenetic relationship of the phages. To identify the phylogenetic relationship among Staphylococcus phages of the Myoviridae family, other S. aureus phages, including Podoviridae (including Staphylococcus phages 44AHJD, 66, GRCS, P68, PT1028, S24-1, and SAP-2) and Siphoviridae (including Staphylococcus phages 3MRA, 13, 29, 52A, 55, 69, 77, 92, 187, 2638A, DW2, IME-SA4, Ipla7, P954, phi5967PVL, phiSa119, phiSauS-IPLA88, PVL, SA13, SA97, SAP-26, SpaA1, StauST-398-3, StB20, StB20-like, StB27, 3A, 11, 23MRA, 37, 71, 88, 96, B166, B236, CNPH82, Ipla5, JS01, phiBU01, phiETA, phiETA2, phiETA3, phiJB, phiMR11, phiNM1, and phiNM2) (phages' nucleic acid access numbers refer additional file 1), were chosen to evaluate the phylogenetic relationship using complete genome sequences (http://www.ncbi.nlm.nih.gov/genome/ viruses/). The neighbour-joining tree was constructed using MEGA $5^{19}$.

\section{Results}

General genomic features of Staphylococcus phages of the Myoviridae family. A total of 22 Staphylococcus phages with complete genome sequence data were examined: K, G1, Twort, A5W, Sb-1, ISP, SA5, GH15, JD007, SA11,vB_SauM_Remus, vB_SauM_Romulus, S25-3, S25-4, phiIPLA-RODI, phiIPLA-C1C, phiSA012, Team1, P108, MCE-2014, 812, and SA1 ${ }^{7-15}$. The Staphylococcus phages Staphy1N, MSA6, 676Z, P4W, and Fi200w, for which only partial genome sequences were available, were excluded from this study. The phages have been isolated worldwide, and all belong to the Myoviridae family. As shown in Table 1, the genomes were approximately $127 \mathrm{~kb}-148 \mathrm{~kb}$ in length, contained between 170 and 220 ORFs, and had a GC content of 27-31\%. However, the GC content of phage SA1 was $45.83 \%$, which was much higher than other Staphylococcus phages of the Myoviridae family. 


\begin{tabular}{|l|c|c|c|c|c|c|c|}
\hline Phages & Country & Size $(\mathbf{b p})$ & GC $\%$ & Access No. & Lytic/Lysogenic & Date & Refs \\
\hline K & USA & 127395 & 30.60 & NC_005880 & lytic & 2004 & 10 \\
\hline G1 & Georgia & 138715 & 30.39 & AY954969.1 & lytic & 2005 & 33 \\
\hline Twort & Canada & 130706 & 30.26 & NC_007021 & lytic & 2005 & 33 \\
\hline A5W & Poland & 137083 & 30.47 & EU418428.1 & lytic & 2008 & NCBI \\
\hline Sb-1 & Georgia & 127188 & 30.48 & HQ163896.1 & lytic & 2011 & 34 \\
\hline ISP & Georgia & 138339 & 30.42 & FR852584.1 & lytic & 2011 & 7 \\
\hline SA5 & Georgia & 137031 & 30.42 & JX875065 & lytic & 2012 & NCBI \\
\hline GH15 & China & 139806 & 30.23 & JQ686190 & lytic & 2012 & 14 \\
\hline JD007 & China & 141836 & 30.37 & JX878671 & lytic & 2012 & 13 \\
\hline SA11 & Korea & 136326 & 30.04 & JX194239 & lytic & 2012 & 8 \\
\hline vB_SauM_Remus & Belgium & 134643 & 29.97 & JX846612 & lytic & 2013 & 15 \\
\hline vB_SauM_Romulus & Belgium & 131332 & 30.01 & JX846613 & lytic & 2013 & 15 \\
\hline S25-3 & Japan & 139738 & 30.22 & AB853330 & lytic & 2013 & 9 \\
\hline S25-4 & Japan & 132123 & 30.31 & AB853331 & lytic & 2013 & 9 \\
\hline phiIPLA-RODI & Belgium & 142348 & 30.42 & KP027446 & lytic & 2015 & 11 \\
\hline phiIPLA-C1C & Belgium & 140961 & 27.98 & KP027447 & lytic & 2015 & 11 \\
\hline phiSA012 & Japan & 142094 & 30.31 & NC_023573 & lytic & 2009 & 35 \\
\hline Team1 & Canada & 140903 & 30.33 & KC012913.1 & lytic & 2014 & 36 \\
\hline P108 & China & 140807 & 30.22 & KM216423.1 & lytic & 2014 & NCBI \\
\hline MCE-2014 & U.K. & 141907 & 30.38 & NC_025416 & lytic & 2014 & 37 \\
\hline 812 & USA & 142096 & 30.40 & NC_029080 & lytic & 2016 & NCBI \\
\hline SA1 & USA & 147303 & 45.83 & NC_027991 & lytic & 2015 & NCBI \\
\hline
\end{tabular}

Table 1. The Staphylococcus phages of the Myoviridae family with published complete genome sequences (through June, 2016).

Lytic or lysogenic analysis. PHACTS, a computational approach used to classify the lifestyle of bacteriophages, was used after the complete genome sequences were annotated and the proteins were converted into the FASTA format. The results showed that the Staphylococcus phages K, G1, Twort, A5W, Sb-1, ISP, SA5, GH15, JD007, SA11,vB_SauM_Remus, vB_SauM_Romulus, S25-3, S25-4, phiIPLA-RODI, phiIPLA-C1C, phiSA012, Team1, P108, MCE-2014, 812, and SA1 belong to the Myoviridae family and are all lytic phages ${ }^{7-15}$. The previous report shows that phage phiIPLA-RODI and phiIPLA-C1C are lytic phages ${ }^{11}$, our results were consistent with the studies reported previously.

Genome organization and annotation. The complete genomic sequences of Staphylococcus phages of the Myoviridae family were compared. As showed in Fig. 1, the genomic structure was chimeric and linear. Several functional modules shown in Table 2 consistently exhibited mosaicism in the genomes, including those involved in packaging, lysis, structure, phage and host interactions, DNA manipulation, and some ORFs with additional functions. High degrees of sequence similarity were evident across the phage sequences, which is unique among Staphylococcus phages of the Myoviridae family. While, recombinase gene was widely existed in the complete genomes of Staphylococcus phages of the Myoviridae family, what suggest that genomic sequences combination may occur between the homologue sequences during phage infection. The difficulties to knock out recombinase gene in these phages may block experimental validation the fully inactivity of Recombinase or confirmation there were no homologue sequences combination occur in the recombinase knock-out phages.

Analysis of virulence and antibiotic resistance genes. All of the predicted ORFs were compared with the sequences in the Antibiotic Resistance Genes Database (ARDB) and the virulence factors database VFDB using the BLASTn algorithm, with the criteria that genes with more than $70 \%$ coverage and $30 \%$ identity were considered to exhibit positive results. No significant hits based on the ORFs predicted in the complete genomes of the Staphylococcus phages of the Myoviridae family were obtained.

As shown in Fig. 2, the SceD-like transglycosylase, which is a biomarker for vancomycin-intermediate strains $^{24}$, was encoded in the genome of Staphylococcus phages K, G1, Twort, A5W, Sb-1, ISP, SA5, GH15, JD007, SA11, vB_SauM_Remus, vB_SauM_Romulus, S25-3, S25-4, phiIPLA-RODI, phiIPLA-C1C, phiSA012, Team1, P108, MCE-2014, and $812^{7-15}$. Furthermore, approximately $70 \%$ of the ORFs were predicted to have unknown functions. It suggested that these ORFs encoding proteins with no similarity functional domains of proteins in InterProScan and CDD database.

Clustering of Staphylococcus phages of the Myoviridae family. The phylogeny tree showed that Staphylococcus phages of the Myoviridae family clustered in the same branch, based on the alignments of the complete genome sequences. As shown in Fig. 3, the phages K, G1, Twort, A5W, Sb-1, ISP, SA5, GH15, JD007, SA11, vB_SauM_Remus, vB_SauM_Romulus, S25-3, S25-4, phiIPLA-RODI, phiIPLA-C1C, phiSA012, Team1, P108, MCE-2014, 812, and SA $1^{7-15}$ clustered together on the same sub-branch with other Staphylococcus phages of the Myoviridae family. These results are consistent with the morphology of the phages. It is well known that 


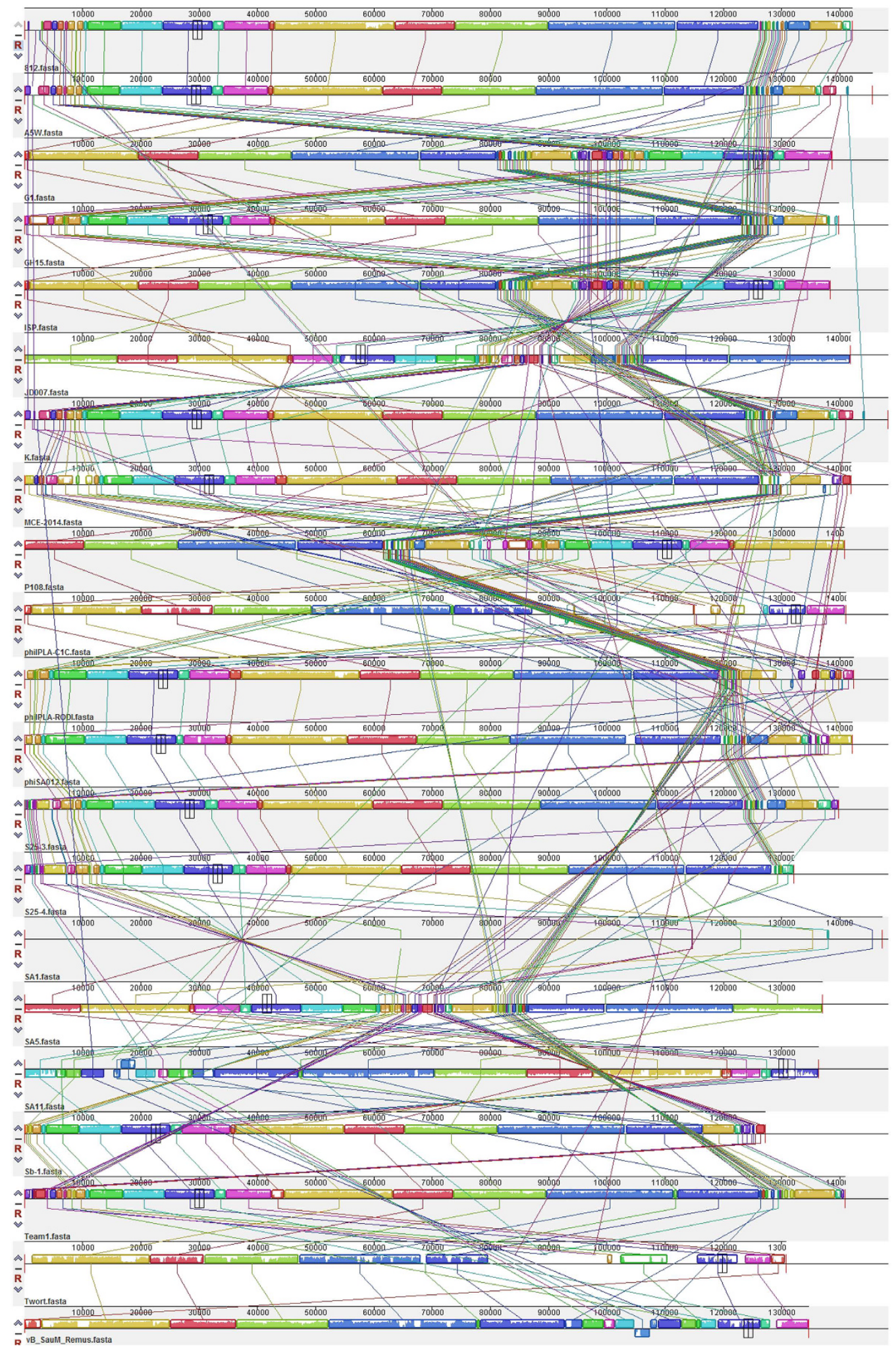

Figure 1. Comparative genomic analysis of Staphylococcus phages of the Myoviridae family.

the proteins such as Helicase, DNA Polymerase, Primase, Large terminase and Major Capsid Protein encoded in the genome of phages are usually chosen to draw phylogenic trees ${ }^{25}$. However, such genes are not always simultaneously existed in the genomes of phages our study enrolled, so it is difficult to cluster phages using these genes when some phages have while others are not. With the development of genome sequencing recently, the complete genome sequences of viruses with high identity were chosen to draw the phylogenic trees with high accuracy. 


\begin{tabular}{|c|c|c|c|c|c|c|c|c|c|c|c|c|c|c|c|c|c|c|c|c|c|c|c|}
\hline Modules & $\begin{array}{l}\text { Functional } \\
\text { ORFs }\end{array}$ & 812 & A5W & G1 & GH15 & ISP & JD007 & $\mathbf{K}$ & $\begin{array}{l}\text { MCE- } \\
2014\end{array}$ & P108 & \begin{tabular}{|l|} 
phiI- \\
PLA- \\
C1C \\
\end{tabular} & $\begin{array}{c}\text { phiIPLA } \\
\text {-ROD1 }\end{array}$ & $\begin{array}{c}\text { phiSA } \\
012\end{array}$ & S25-3 & S25-4 & SA11 & Twort & Sb-1 & Team1 & SA5 & 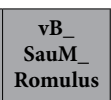 & \begin{tabular}{|l|}
$\mathrm{vB}_{-}$ \\
SauM_- \\
Remus
\end{tabular} & SA1 \\
\hline \multirow{4}{*}{ Replication } & $\begin{array}{l}\text { DNA } \\
\text { helicase }\end{array}$ & + & + & + & + & + & + & + & + & + & + & + & + & + & + & + & + & + & + & + & + & + & + \\
\hline & \begin{tabular}{|l|} 
DNA \\
polymerase \\
I
\end{tabular} & + & + & + & + & + & + & + & + & + & + & + & + & + & + & + & + & + & + & + & + & + & + \\
\hline & \begin{tabular}{|l} 
DNA \\
primase
\end{tabular} & + & + & + & + & + & + & + & + & & + & + & + & + & + & + & + & + & + & + & + & + & + \\
\hline & $\begin{array}{l}\text { Ribonu- } \\
\text { clease } \mathrm{H}\end{array}$ & + & + & + & + & + & + & + & + & + & + & + & + & + & + & + & + & + & + & + & + & + & \\
\hline Introns & $\begin{array}{l}\text { HNH } \\
\text { homing } \\
\text { endonuc- } \\
\text { lease }\end{array}$ & + & + & + & + & + & & + & + & & + & + & + & & & + & + & + & + & + & + & + & + \\
\hline \multirow{2}{*}{ Lysis } & Endolysin & + & + & + & + & + & + & + & + & + & + & + & + & + & + & + & + & + & + & + & + & + & + \\
\hline & Holin & + & + & + & + & + & + & + & + & + & + & + & + & + & + & + & + & + & + & + & + & + & \\
\hline Packaging & \begin{tabular}{|l} 
Terminase, \\
large \\
subunit
\end{tabular} & + & + & + & + & + & + & + & + & + & + & + & + & + & + & + & + & + & + & + & + & + & + \\
\hline \multirow{5}{*}{ Structure } & Baseplate & + & + & + & + & + & + & + & + & + & + & + & + & + & + & + & + & + & + & + & + & + & + \\
\hline & $\begin{array}{l}\text { Capsid and } \\
\text { scaffold }\end{array}$ & + & + & + & + & + & + & + & + & + & + & + & + & + & + & + & + & + & + & + & + & + & \\
\hline & \begin{tabular}{|l|} 
Major \\
capsid \\
protein
\end{tabular} & + & + & + & + & + & + & + & + & + & + & + & + & + & + & & + & + & + & + & + & + & + \\
\hline & $\begin{array}{l}\text { Major tail } \\
\text { protein }\end{array}$ & + & + & + & + & + & + & + & + & + & & + & + & + & + & + & + & + & + & + & + & + & \\
\hline & \begin{tabular}{|l|}
$\begin{array}{l}\text { Major tail } \\
\text { sheath }\end{array}$ \\
\end{tabular} & + & + & + & + & + & + & + & + & + & + & + & + & + & + & + & + & + & + & + & + & + & \\
\hline \multirow{3}{*}{ Regulation } & \begin{tabular}{|l|} 
DNA \\
transfer \\
protein
\end{tabular} & + & + & + & + & + & + & + & + & + & + & + & & + & + & + & & + & + & + & + & + & \\
\hline & \begin{tabular}{|l|} 
DNA- \\
binding \\
protein \\
\end{tabular} & + & + & + & + & + & + & + & + & + & + & + & & + & + & + & & + & + & + & + & + & \\
\hline & $\begin{array}{l}\text { Integration } \\
\text { host factor }\end{array}$ & + & + & + & + & + & + & + & + & + & + & + & + & + & + & + & + & + & + & + & + & + & \\
\hline \multirow{4}{*}{$\begin{array}{l}\text { Recomb- } \\
\text { ination }\end{array}$} & \begin{tabular}{|l} 
Recomb- \\
inase
\end{tabular} & & & & + & & + & & + & & + & & + & & & + & + & & + & & + & + & \\
\hline & \begin{tabular}{|l|} 
Exonuclease \\
\end{tabular} & + & + & + & + & + & + & + & + & + & + & + & + & + & + & + & + & + & + & + & + & + & + \\
\hline & $\begin{array}{l}\text { Endon- } \\
\text { uclease }\end{array}$ & + & + & + & + & + & + & + & + & + & & + & + & + & + & + & + & + & + & + & + & + & \\
\hline & \begin{tabular}{|l|} 
RecA \\
protein
\end{tabular} & + & + & + & & + & & + & & + & & + & & + & + & & & + & & + & & & \\
\hline
\end{tabular}

Table 2. The functional modules of the ORFs in the genomes of Staphylococcus phages of the Myoviridae family.

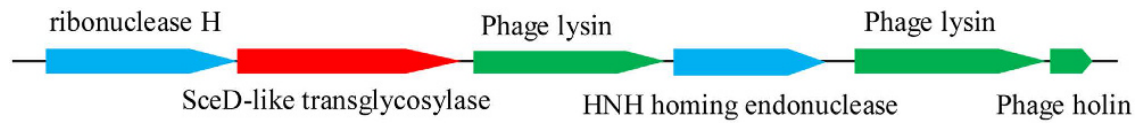

Figure 2. The SceD-like transglycosylase gene is widely distributed in the genome of Staphylococcus phages of the Myoviridae family.

\section{Discussions}

Phage therapy has become a subject of renewed interest recently, and some phages have been used in clinical trials, including the Staphylococcus phages of the Myoviridae family. Several studies have evaluated the safety of certain phages in clinical trials. Rhoads et al. reported a study in which ulcers were treated for 12 weeks with bacteriophages targeting Pseudomonas aeruginosa, S. aureus, and Escherichia coli or a saline control ${ }^{3}$. Follow-up continued until week 24 , and no adverse events were attributed to the phage therapy. In addition, no significant difference was determined between the test and control groups regarding the frequency of adverse events, rate of healing, or frequency of healing ${ }^{3}$. Unlike normal antibiotics, a bacteriophage is a type of virus that contains genetic material. Therefore, potentially useful phages require full evaluation prior to use in phage therapy. A bacteriophage may be a potential mechanism for the transmission of antibiotic resistance genes or virulence genes among bacteria. A prophage may contribute the pathogenic traits of Enterococcus feaclis ${ }^{26}$. Antibiotic resistance 


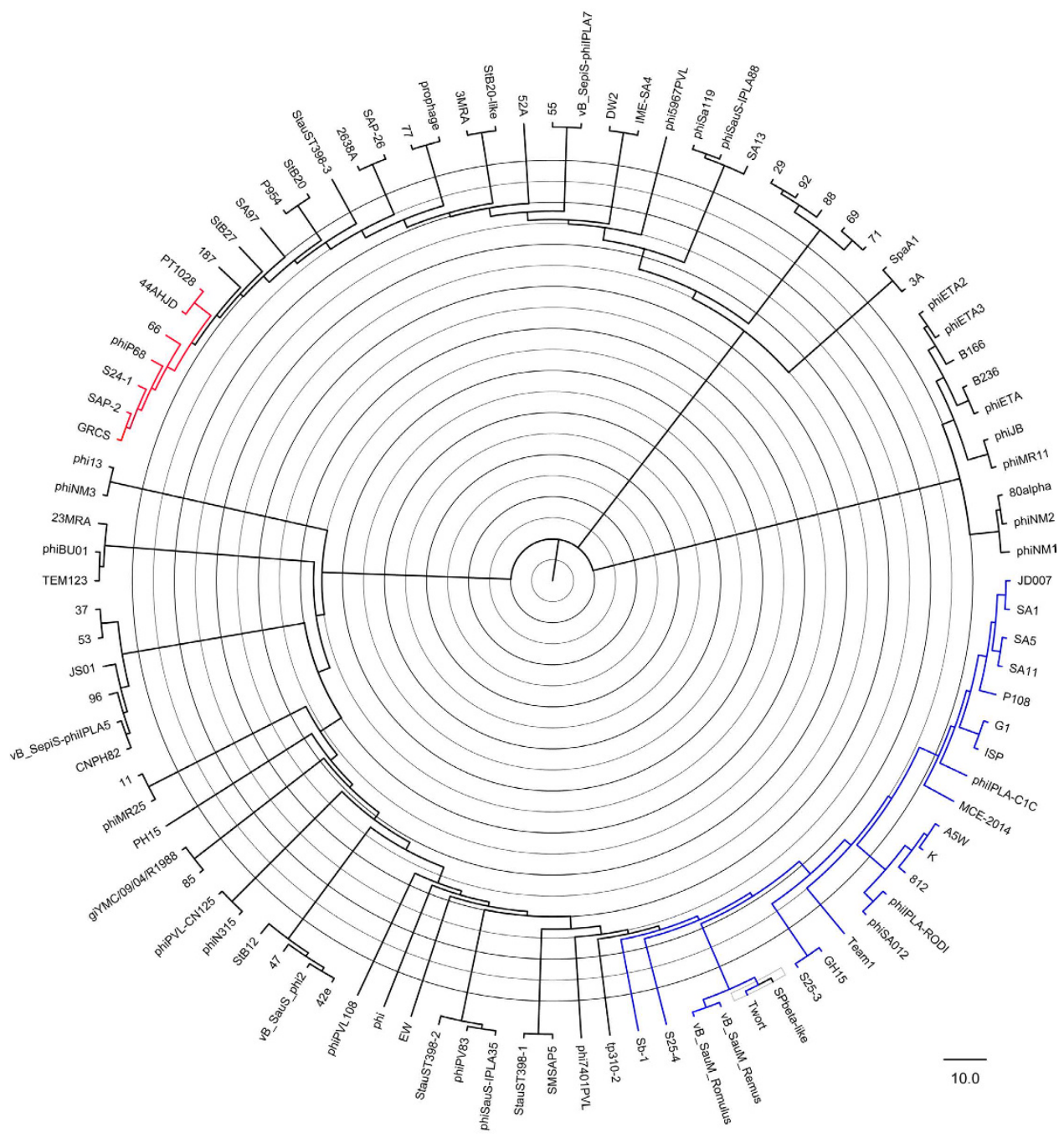

Figure 3. The phylogenetic tree of Staphylococcus phages of the Myoviridae family. The diagram represents the phylogeny constructed using the complete phage genome sequences via the neighbour-joining method.

genes, including blaTEM, qnrA, blaCTX-M-1, and toxin genes, have been found in the DNA of bacteriophages isolated from human faecal samples ${ }^{27,28}$. Therefore, safety assessment of phages at the genome level is critically important. First, an analysis should determine whether the bacteriophage carries genes that may accelerate the virulence and antibiotic resistance of bacteria when these genes integrate into their genomes. Second, the ability of the bacteriophage genome that will integrate into the genome of bacteria should be assessed. In this evaluation, it is important and necessary to assess the safety of bacteriophages at the genome level. McCallin et al. evaluated the safety of a phage cocktail from one company, and a small volunteer trial did not report an association between adverse effects and oral phage exposure ${ }^{29}$. During that study, the genome sequences of phages were obtained, and bioinformatics analysis revealed that no undesirable genes were present ${ }^{29}$. A safety assessment of the phage at the genome level is an essential step during the evaluation of the suitability of phages for therapeutic applications.

Staphylococcus phages of the Myoviridae family are potentially useful for phage therapy. Dozens of phages have been shown to protect mice from death after infection with $S$. aureus. Some phages have been used in clinical trials, and no adverse effects have been reported. In this report, our results showed that none of these bacteriophages carried any antibiotic resistance or virulence genes. In addition, all of the studied bacteriophages were virulent and were therefore unable to integrate into the genome of $S$. aureus. Our studies show that Staphylococcus phages of the Myoviridae family are safe at the genome level. While, as showed in Table 2, recombinase was widely distributed in phage GH15, JD007, MCE-2014, phiIPLA-C1C, phiSA012, SA11, Twort. Team1, vB_SauM_Romulus and vB_SauM_Remus, it was reported that Recombinase could recombine homologous genome sequences between bacteriophages or/and its host ${ }^{30,31}$; and the SceD-like transglycosylase, which is a biomarker for vancomycin-intermediate strains ${ }^{24}$ is encoded in the genome of Staphylococcus phages K, G1, Twort, A5W, Sb-1, ISP, SA5, GH15, JD007, SA11, vB_SauM_Remus, vB_SauM_Romulus, S25-3, S25-4, phiIPLA-RODI, phiIPLA-C1C, phiSA012, Team1, P108, MCE-2014, and $812^{7-15}$. The expression of this gene during S. aureus infection may increase the minimum inhibitory concentration of vancomycin used for the treatment of infections caused by MRSA; furthermore, approximately $70 \%$ of the ORFs encoded in the phage genomes have unknown 
functions, and their potential roles in S. aureus antibiotic resistance and virulence are unknown, what's more, with the development of genome sequencing, acquisition of a complete genome sequence of organism become much more easily, and lots of ORFs encoded proteins with unknown functions ${ }^{32}$. All of the above factors represent a potential genome-level risk for phage therapy. Therefore, the clinical use of these phages should be evaluated comprehensively.

In summary, Staphylococcus phages of the Myoviridae family have been isolated worldwide. Morphology studies have shown that these phages are contractile between the head and tail. In addition, they have a wide host range and can prevent infectious diseases in animal models, and pre-clinical and clinical trials have not reported any adverse effects. Our results show that Staphylococcus phages of the Myoviridae family and are lytic and therefore cannot integrate into the genomes of their host. In addition, no antibiotic resistance or virulence genes were present in their genomes, indicating that they are safe at the genome level. In addition, the phages analyzed in this study clustered in the same branch of an evolutionary tree, suggesting that they share a common ancestor.

\section{References}

1. Wright, A., Hawkins, C. H., Anggard, E. E. \& Harper, D. R. A controlled clinical trial of a therapeutic bacteriophage preparation in chronic otitis due to antibiotic-resistant Pseudomonas aeruginosa; a preliminary report of efficacy. Clin Otolaryngol 34, 349-357, doi: COA1973.

2. Bruttin, A. \& Brussow, H. Human volunteers receiving Escherichia coli phage T4 orally: a safety test of phage therapy. Antimicrob Agents Chemother 49, 2874-2878, doi: 49/7/2874.

3. Rhoads, D. D. et al. Bacteriophage therapy of venous leg ulcers in humans: results of a phase I safety trial. J Wound Care 18, 237-238, 240-233, doi: 10.12968/jowc.2009.18.6.42801 (2009)

4. Chen, J. \& Novick, R. P. Phage-mediated intergeneric transfer of toxin genes. Science 323, 139-141, doi: 323/5910/139.

5. Granobles Velandia, C. V., Kruger, A., Parma, Y. R., Parma, A. E. \& Lucchesi, P. M. Differences in Shiga toxin and phage production among stx(2g)-positive STEC strains. Front Cell Infect Microbiol 2, 82, doi: 10.3389/fcimb.2012.00082 (2012).

6. Colomer-Lluch, M., Imamovic, L., Jofre, J. \& Muniesa, M. Bacteriophages carrying antibiotic resistance genes in fecal wastes from cattle, pigs and poultry. Antimicrob Agents Chemother, doi: AAC.00535-11.

7. Vandersteegen, K. et al. Microbiological and molecular assessment of bacteriophage ISP for the control of Staphylococcus aureus. PLoS One 6, e24418, doi: 10.1371/journal.pone.0024418PONE-D-11-09572.

8. Kim, M. S. \& Myung, H. Complete Genome of Staphylococcus aureus Phage SA11. J Virol 86, 10232, doi: 86/18/10232.

9. Takemura-Uchiyama, I. et al. Genomic and phylogenetic traits of Staphylococcus phages S25-3 and S25-4 (family Myoviridae, genus Twort-like viruses). Ann Microbiol (Paris), doi: 10.1007/s13213-013-0762-2 (2013).

10. O'Flaherty, S. et al. Genome of staphylococcal phage K: a new lineage of Myoviridae infecting gram-positive bacteria with a low $\mathrm{G}+\mathrm{C}$ content. Journal of bacteriology 186, 2862-2871, doi: 10.1128/JB.186.9.2862-2871 (2004).

11. Gutierrez, D. et al. Two Phages, phiIPLA-RODI and phiIPLA-C1C, Lyse Mono- and Dual-Species Staphylococcal Biofilms. Appl Environ Microbiol 81, 3336-3348, doi: 10.1128/AEM.03560-14 (2015).

12. Lobocka, M. et al. Genomics of Staphylococcal Twort-like Phages - Potential Therapeutics of the Post-Antibiotic Era. Adv Virus Res 83, 143-216, doi: B978-0-12-394438-2.00005-0.

13. Cui, Z. et al. Complete Genome Sequence of Wide-Host-Range Staphylococcus aureus Phage JD007. J Virol 86, 13880-13881, doi: 10.1128/JVI.02728-12 (2012).

14. Gu, J. et al. Complete Genome Sequence of Staphylococcus aureus Bacteriophage GH15. J Virol 86, 8914-8915, doi: 86/16/8914.

15. Vandersteegen, K. et al. Romulus and Remus, two phage isolates representing a distinct clade within the Twortlikevirus genus, display suitable properties for phage therapy applications. J Virol 87, 3237-3247, doi: 10.1128/JVI.02763-12 (2013).

16. Takemura-Uchiyama, I. et al. Evaluating efficacy of bacteriophage therapy against Staphylococcus aureus infections using a silkworm larval infection model. FEMS Microbiol Lett., doi: 10.1111/1574-6968.12220 (2013).

17. McDowall, J. \& Hunter, S. InterPro protein classification. Methods Mol Biol 694, 37-47, doi: 10.1007/978-1-60761-977-2_3 (2011).

18. Marchler-Bauer, A. et al. CDD: a Conserved Domain Database for the functional annotation of proteins. Nucleic Acids Res 39, D225-229, doi: 10.1093/nar/gkq1189.

19. Tamura, K. et al. MEGA5: molecular evolutionary genetics analysis using maximum likelihood, evolutionary distance, and maximum parsimony methods. Molecular biology and evolution 28, 2731-2739, doi: 10.1093/molbev/msr121 (2011).

20. Rissman, A. I. et al. Reordering contigs of draft genomes using the Mauve aligner. Bioinformatics 25, 2071-2073, doi: 10.1093/ bioinformatics/btp356 (2009)

21. McNair, K., Bailey, B. A. \& Edwards, R. A. PHACTS, a computational approach to classifying the lifestyle of phages. Bioinformatics 28, 614-618, doi: 10.1093/bioinformatics/bts014 (2012).

22. Liu, B. \& Pop, M. ARDB-Antibiotic Resistance Genes Database. Nucleic Acids Res 37, D443-447, doi: 10.1093/nar/gkn656 (2009).

23. Zhou, C. E. et al. MvirDB-a microbial database of protein toxins, virulence factors and antibiotic resistance genes for bio-defence applications. Nucleic Acids Res 35, D391-394, doi: 10.1093/nar/gkl791 (2007).

24. Drummelsmith, J., Winstall, E., Bergeron, M. G., Poirier, G. G. \& Ouellette, M. Comparative proteomics analyses reveal a potential biomarker for the detection of vancomycin-intermediate Staphylococcus aureus strains. Journal of proteome research 6, 4690-4702, doi: $10.1021 /$ pr070521m (2007).

25. El-Arabi, T. F. et al. Genome sequence and analysis of a broad-host range lytic bacteriophage that infects the Bacillus cereus group. Virol J 10, 48, doi: 10.1186/1743-422X-10-48 (2013).

26. Matos, R. C. et al. Enterococcus faecalis prophage dynamics and contributions to pathogenic traits. PLoS genetics 9, e1003539, doi: 10.1371/journal.pgen.1003539 (2013).

27. Quiros, P. et al. Antibiotic resistance genes in the bacteriophage DNA fraction of human fecal samples. Antimicrob Agents Chemother 58, 606-609, doi: 10.1128/AAC.01684-13 (2014).

28. Martinez-Castillo, A., Quiros, P., Navarro, F., Miro, E. \& Muniesa, M. Shiga toxin 2-encoding bacteriophages in human fecal samples from healthy individuals. Appl Environ Microbiol 79, 4862-4868, doi: 10.1128/AEM.01158-13 (2013).

29. McCallin, S. et al. Safety analysis of a Russian phage cocktail: from metagenomic analysis to oral application in healthy human subjects. Virology 443, 187-196, doi: 10.1016/j.virol.2013.05.022 (2013).

30. Yang, P., Wang, J. \& Qi, Q. Prophage recombinases-mediated genome engineering in Lactobacillus plantarum. Microb Cell Fact 14, 154, doi: 10.1186/s12934-015-0344-z (2015).

31. De Paepe, M. et al. Temperate phages acquire DNA from defective prophages by relaxed homologous recombination: the role of Rad52-like recombinases. PLoS Genet 10, e1004181, doi: 10.1371/journal.pgen.1004181 (2014).

32. Hoai, T. D., Nishiki, I. \& Yoshida, T. Properties and genomic analysis of Lactococcus garvieae lysogenic bacteriophage PLgT-1, a new member of Siphoviridae, with homology to Lactococcus lactis phages. Virus Res 222, 13-23, doi: 10.1016/j.virusres.2016.05.021 (2016).

33. Kwan, T., Liu, J., DuBow, M., Gros, P. \& Pelletier, J. The complete genomes and proteomes of 27 Staphylococcus aureus bacteriophages. Proc Natl Acad Sci USA 102, 5174-5179, doi: 10.1073/pnas.0501140102. 
34. Kvachadze, L. et al. Evaluation of lytic activity of staphylococcal bacteriophage Sb-1 against freshly isolated clinical pathogens. Microbial biotechnology 4, 643-650, doi: 10.1111/j.1751-7915.2011.00259.x (2011).

35. Synnott, A. J. et al. Isolation from sewage influent and characterization of novel Staphylococcus aureus bacteriophages with wide host ranges and potent lytic capabilities. Appl Environ Microbiol 75, 4483-4490, doi: 10.1128/AEM.02641-08 (2009).

36. El Haddad, L. et al. Improving the safety of Staphylococcus aureus polyvalent phages by their production on a Staphylococcus xylosus strain. PLoS One 9, e102600, doi: 10.1371/journal.pone.0102600 (2014).

37. Alves, D. R. et al. Combined use of bacteriophage K and a novel bacteriophage to reduce Staphylococcus aureus biofilm formation. Appl Environ Microbiol 80, 6694-6703, doi: 10.1128/AEM.01789-14 (2014).

\section{Acknowledgements}

This work was sponsored in part by the National Natural Science Foundation of China (No. 31500154), the Shanghai Sailing Program (No. 15YF1409500), the fund of the Shanghai Health and Family Planning Committee (No. 201440289), the Pre-outstanding Medical Youth Program of Shanghai General Hospital (No. 06N1503012), and the Shanghai Jiao Tong University School of Medicine Dr Innovation Fund (No. BXJ201201).

\section{Author Contributions}

Z.L.C. and X.K.G. performed the molecular genetic studies, participated in the sequence alignment, and drafted the manuscript. Q.T.L., K.D., L.B.Z., Y.Z., R.T. and Y.Z.Z. participated in the sequence alignment. Z.L.C. and L.L. participated in the design of the study and performed the statistical analysis. Z.L.C. and L.L. conceived of the study, participated in the study design and coordination, and helped to draft the manuscript.

\section{Additional Information}

Supplementary information accompanies this paper at http://www.nature.com/srep

Competing financial interests: The authors declare no competing financial interests.

How to cite this article: Cui, Z. et al. Safety assessment of Staphylococcus phages of the family Myoviridae based on complete genome sequences. Sci. Rep. 7, 41259; doi: 10.1038/srep41259 (2017).

Publisher's note: Springer Nature remains neutral with regard to jurisdictional claims in published maps and institutional affiliations.

(c) (i) This work is licensed under a Creative Commons Attribution 4.0 International License. The images or other third party material in this article are included in the article's Creative Commons license, unless indicated otherwise in the credit line; if the material is not included under the Creative Commons license, users will need to obtain permission from the license holder to reproduce the material. To view a copy of this license, visit http://creativecommons.org/licenses/by/4.0/

(C) The Author(s) 2017 